Holger Maune*, Matthias Jost, Alex Wiens, Christian Weickhmann, Roland Reese, Mohammad Nikfalazar, Christian Schuster, Tobias Franke, Wenjuan Hu, Matthias Nickel, Daniel Kienemund, Ananto Eka Prasetiadi and Rolf Jakoby

\title{
Tunable Microwave Component Technologies for SatCom-Platforms
}

DOI 10.1515/freq-2016-0207

Received July 4, 2016

\begin{abstract}
Modern communication platforms require a huge amount of switched RF component banks especially made of different filters and antennas to cover all operating frequencies and bandwidth for the targeted services and application scenarios. In contrast, reconfigurable devices made of tunable components lead to a considerable reduction in complexity, size, weight, power consumption, and cost. This paper gives an overview of suitable technologies for tunable microwave components especially for SatCom applications. Special attention is given to tunable components based on functional materials such as barium strontium titanate (BST) and liquid crystal (LC).
\end{abstract}

Keywords: tunable components, agile systems, ferroelectrics, liquid crystal

\section{Introduction}

A large need for tunable components exists in SatCom applications for realization of reconfigurable systems. Possible applications are beam-steering or -forming antennas, e. $\mathrm{g}$. enabling an uplink from a low earth orbit satellite to a relay satellite in a geostationary orbit. These inter-satellite links can be realized by beam-steering antennas, where the main beam of the antenna is adaptively pointed to the target of interest. Also beam-forming applications with variable antenna footprints are a common task. Figure 1 gives an overview of possible application scenarios. Not only the antenna part of the platform needs to be adaptive in future

*Corresponding author: Holger Maune, Institute for Microwave Engineering and Photonics, Technische Universität Darmstadt, Darmstadt, Germany, E-mail: maune@imp.tu-darmstadt.de Matthias Jost, Alex Wiens, Christian Weickhmann, Roland Reese, Mohammad Nikfalazar, Christian Schuster, Tobias Franke, Wenjuan Hu, Matthias Nickel, Daniel Kienemund, Ananto Eka Prasetiadi, Rolf Jakoby: E-mail: jakoby@imp.tu-darmstadt.de, Institute for Microwave Engineering and Photonics, Technische Universität Darmstadt, Darmstadt, Germany systems, but also the RF frontend itself benefits from the development of tunable components. Since many years, these components are of major interest for next generation communication systems, especially for terrestrial communication. Examples are frequency agile components for mobile communications below $10 \mathrm{GHz}$. The development for $5 \mathrm{G}$ communication systems e. g. in the $60 \mathrm{GHz}$. band converges more and more with the requirements set by satellite communications. Also in satellite platforms, the operator wants to have the opportunity to change for example the band and mode of operation while the satellite is operational. Hence, the communication systems of the future will merge together, as the VDE ITG recently concluded in [1]. This results in the fact that also flying platform will require the "ultimate" RF frontends [2] shown in Figure 2.

The major advantage of reconfigurable systems, beside the adaptation to different communication scenarios, is a reduction in complexity and size. This leads to low-weight and energy-efficient components that are required by satellite operators in particular. The proposed frontend and antenna system consist of numerous tunable RF components, implementing a frequency- and/or impedance-variable behavior, such as phase shifters, adaptive impedance matching networks, and tunable filters, as outlined in Figure 2. For the realization of such components, different technologies can be applied. In the following section suitable technologies will be introduced and compared. After this, different application scenarios will be presented.

\section{Technologies for tunable components}

There are many different technologies available for the realization of tunable microwave components. These can be grouped into three categories: (1) electronically and (2) mechanically tuned systems on the one hand and (3) functional materials on the other hand. The most commonly used technology is the semiconductor technology, due to the high volume market and acceptable 


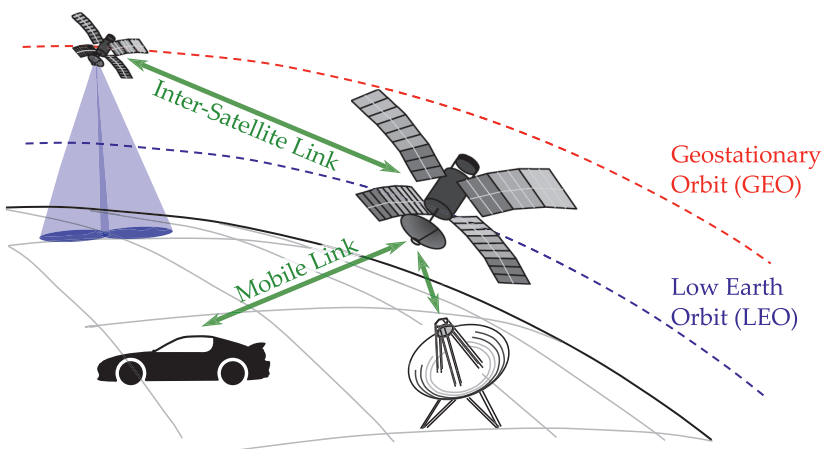

Figure 1: Different application scenarios for beam-steering and -forming antennas on a satellite platform.

performance. For many new and upcoming applications the performance, such as linearity and power consumption, is insufficient. Here, more modern approaches such as micro-electro-mechanical systems (MEMS) and functional materials come into play, having unique properties for specific applications. Up to now, there is no technology available which can fulfill all application's requirements. The main requirements from a system engineer's perspective are mainly: addressable frequency range, required bias voltages/power, and tuning speed.

\subsection{Semiconductor technologies}

Based on semiconductor technologies, varactor diodes and PIN-diodes can be used to build tunable capacitance and switches, respectively. The depletion zone of the diode is modulated in terms of an electrical field, resulting in a tuning of the junction capacitance. Semiconductor diodes show mainly two disadvantages: due to the low tuning voltage of a few volt, the RF field is modulating the junction capacitance in the same way as the tuning voltage, resulting in a strong non-linear behavior of the component. PIN-diodes can be used as switches in power applications. Nevertheless, the tuning voltages can easily reach $100 \mathrm{~V}$. In addition, the tuning requires a current flowing through the diode, which increases the power consumption of the circuit even in steady state and might hinder the implementation in mobile and battery powered devices, where the power budget is restricted.

\subsection{Micro-electro-mechanical systems (MEMS)}

In recent years, micro-electro-mechanical systems (MEMS) gained a lot of interest from industry. One major advantage of this technologyis their integrability into common semicondor processes. The tuning of capacitance is realized by changing geometric parameters e. g. distance of two electrodes. These mechanical processes usually require high actuation voltages in the range from $50 \mathrm{~V}$ up to $200 \mathrm{~V}$. One of the major tasks was, and still is, the improvement of the reliability of the mechanical systems, which can be operated also with moderate to high power levels. For satellite communications, the major drawbacks are mechanical reliability and aging of the components, because the elements cannot be replaced once in operation.

\subsection{Ferrites}

Ferrite materials can be tuned by external magnetic fields. While this technology is feasible in highest-power applications, it cannot be used in mobile, handheld and solarpowered applications. The power consumption for the generation of an external magnetic bias field is too high for commonly intended applications in communications.

\subsection{Ferroelectric materials}

Components implementing ferroelectric materials use the field-dependent material's polarization in order to realize a tunable capacitance. As for semiconductor devices, the

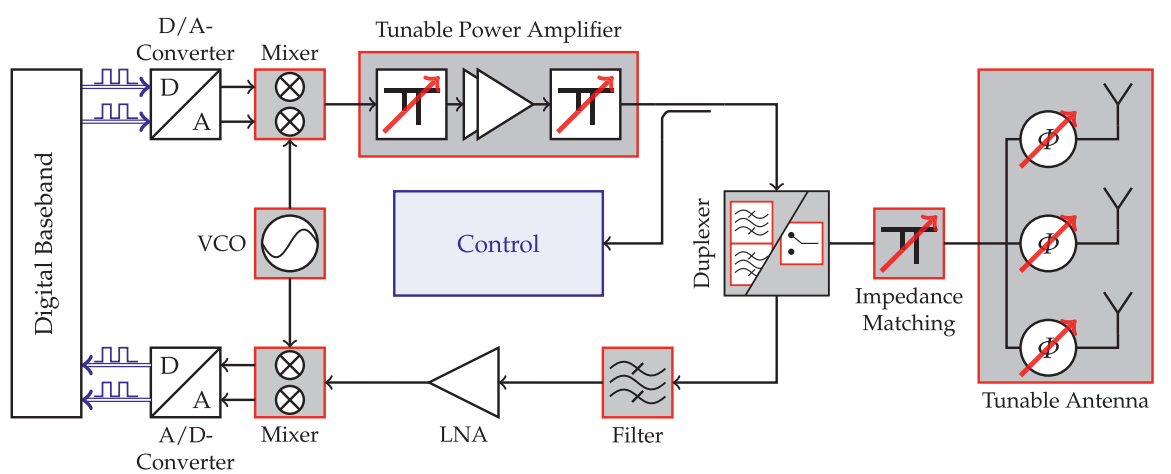

Figure 2: Block diagram of an adaptive transceiver, consisting of a reconfigurable digital base band processor and several tunable components (highlighted in gray). 


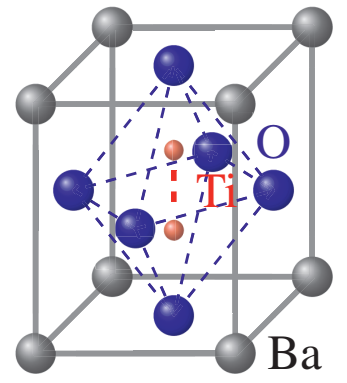

(a) Ferroelectric: tetragonal structure

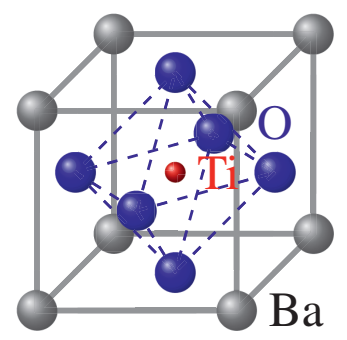

(b) Paraelectric: cubic structure
Figure 3: Crystal lattice of BST (left) below and (right) above the Curie temperature. In the tetragonal structure two energetic identical energy states exists, resulting in the well-known hysteresis of ferroelectric materials. There is only one energetic optimum in the paralectric phase, so no hysteresis occurs.

capacitance can be changed by an electrostatic field applied to the electrodes. The most prominent material in this class is Barium-Strontium-Titanate $\mathrm{Ba}_{x} \mathrm{Sr}_{(1-x)} \mathrm{TiO}_{4}$ (BST). Figure 3 shows the most important crystal configurations of BST for room temperature applications.

Usually, BST components are operated above the Curie temperature $T_{C}$ in order to reduce the losses induced by the hysteresis of the ferroelectric phase. The permittivity $\varepsilon_{r}$ of BST can be described by the model of Vendik et al . [3], which is based on the Ginzburg-Landau theory by

$$
\varepsilon_{r}(E, T)=\frac{\varepsilon_{00}}{\left(\sqrt{\zeta^{2}+v^{3}}+\zeta\right)^{\frac{2}{3}}+\left(\sqrt{\zeta^{2}+v^{3}}-\zeta\right)^{\frac{2}{3}}-v}
$$

with the temperature $(T)$ and field strength $(E)$ dependent quantities

$$
v=\sqrt{\left(\frac{\Theta_{F}}{4 T_{C}}\right)^{2}+\left(\frac{T}{T_{C}}\right)^{2}} \quad \zeta=\sqrt{\left(\frac{E}{E_{N}}\right)^{2}+\xi_{S}^{2}}
$$

and the material parameters $\varepsilon_{00}, \Theta_{F}, E_{N}$, and $\xi_{S}$. The Curie temperature $T_{C}$ can be designed directly by choosing an appropriate ratio $x$ between $\operatorname{Barium} \mathrm{Ba}_{(x)}$ and Strontium $\mathrm{Sr}_{(1-x)}$. The permittivity of a BST bulk ceramic with high crystal quality $\left(\xi_{S}=0.01\right)$ and a thick film with more defects $\left(\xi_{S}=0.15\right)$ is depicted in Figure 4. It can clearly be identified, that the tunability of the material is highest close to the Curie temperature. Unfortunately, the losses are also high at this temperature. As a tradeoff, the material is usually operated at temperatures $20 \mathrm{~K}$ to $30 \mathrm{~K}$ above the Curie temperature. A mixture with $x=60 \%$ Barium exhibits a Curie temperature of $T_{C} \approx-2^{\circ} \mathrm{C}$ and is therefore best suited for room temperature applications. Additionally, the properties of BST can

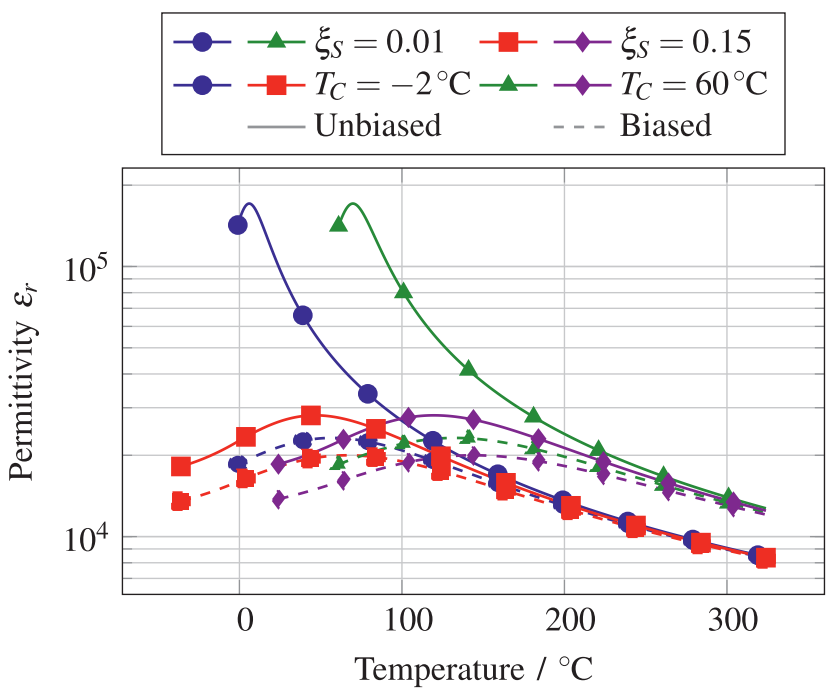

Figure 4: Permittivity of BST according to Vendik (1) for different material qualities $\left(\xi_{S}=0.01\right.$ and $\xi_{S}=0.15$ for bulk ceramics and thick-films, respectively) and different Barium ratios $x$ with different Curie temperatures $T_{C}$. For comparison, the permittivity of the biased (tuned) material is shown with dashed lines.

be adapted by different dopants. Common materials for doping are iron, copper, and fluor [4]. Beside an influence on the Curie temperature, these materials directly affect the tunability as well as the dielectric loss of the material. For comparison of different materials, the material performance parameter $\eta_{\varepsilon}$ is used. It relates the tuning of the permittivity $\tau_{\varepsilon}$ to the maximum dielectric losses $\tan \delta_{\max }$ by

$$
\eta_{\varepsilon}=\frac{\tau_{\varepsilon}(E)}{\tan \delta_{\max }}=\frac{1-\varepsilon_{r}(E) / \varepsilon_{r}(0)}{\tan \delta_{\max }}
$$

Different technologies can be applied for the processing of BST based tunable components. Thin-film components are produced by evaporation methods such as sputtering or pulsed laser deposition (PLD). These films usually have a thickness of less than $500 \mathrm{~nm}$ and show a single or poly-crystalline behavior. Thick-film components are resulting from various printing technologies such as screen printing or ink-jet printing. The poly-crystalline films have a thickness above $1 \mu \mathrm{m}$ after sintering. This sintering step requires a temperature of about $1,250^{\circ} \mathrm{C}$. By implementing composite materials, e.g. by adding $\mathrm{ZnO}-\mathrm{B}_{2} \mathrm{O}_{3}$, this temperature can be reduced to around $800^{\circ} \mathrm{C}$, making BST composite materials compatible to low temperature co-fired ceramics (LTCC) [5]. Measured performance of undoped BST, processed both as bulk ceramic and as thick film is shown in Figure 5. The material performance $\eta_{\varepsilon}$ of the bulk ceramic is higher than that of the thick film. Nevertheless, thick films are better suited for tunable microwave applications. There 

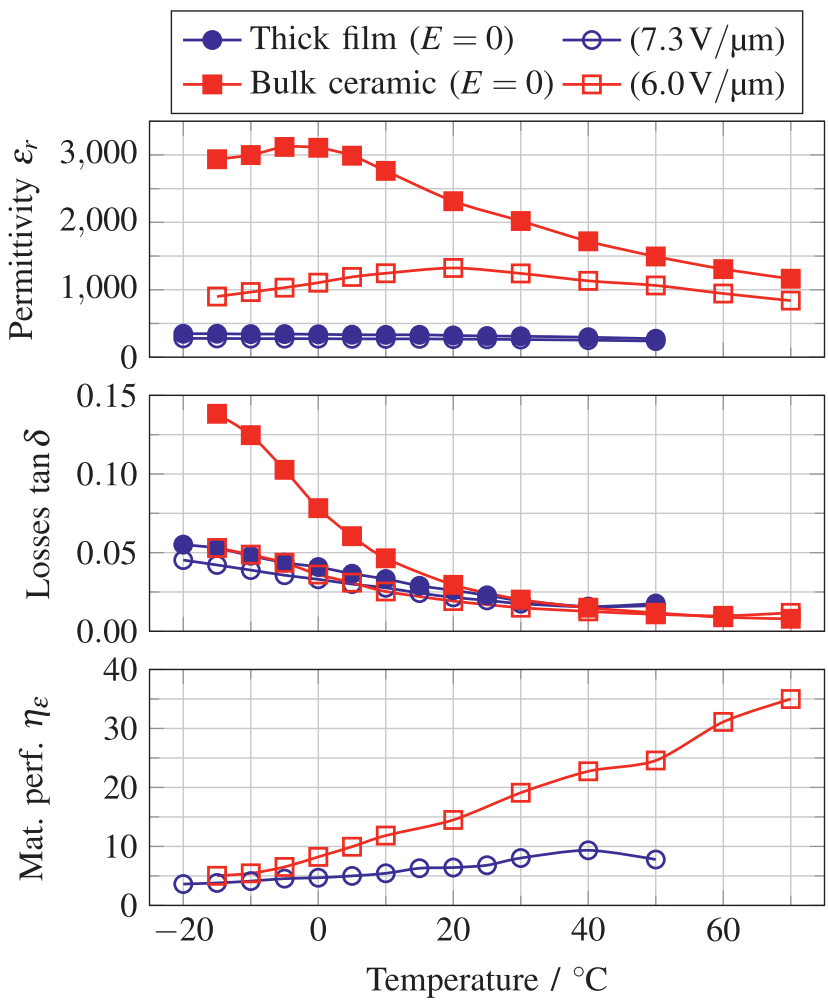

Figure 5: Performance of a $\mathrm{Ba}_{0.6} \mathrm{Sr}_{0.4} \mathrm{TiO}_{3}$ bulk ceramic compared to an undoped thick film at $5 \mathrm{GHz}$ with and without applied tuning field.

are mainly two reasons: (a) the extremely high permittivity of bulk BST ceramics makes the design of microwave components very difficult. For a tunable CPW with low metallic losses the line impedance is in the range of $15 \Omega$ to $30 \Omega$, which increases the effort for impedance matching of the tunable line. In addition, bulk ceramics show acoustic resonances due to induced piezoelectricity comparable to thin films [6], which increases the losses and limits the frequency range of operation. In thick films, those acoustic effects cannot be stimulated in that extend due to the porous micro structure.

Figure 6 shows two different topologies of varactors, which can either be realized in thin- or thick-film technology. Planar inter-digital capacitors (IDC) are mainly used for thick-film components where the metallic electrodes have to be applied after the sintering step. With composite thick films, metal-insulator-metal (MIM) structures could be realized in thick-film technology for the first time [7]. The capacitance of planar varactors can be calculated by conformal mapping methods [8] or by EM simulation tools. For the tunability of the capacitance, the relation of the BST layer thickness $h_{\mathrm{IDC}}$ and gap width $g$ reduces the tunability of the component compared to the material's tunability. It has been proven that $h_{\mathrm{IDC}} \approx g / 5$ is an optimal solution, resulting in a tuning

\section{Planar (IDC) geometry}
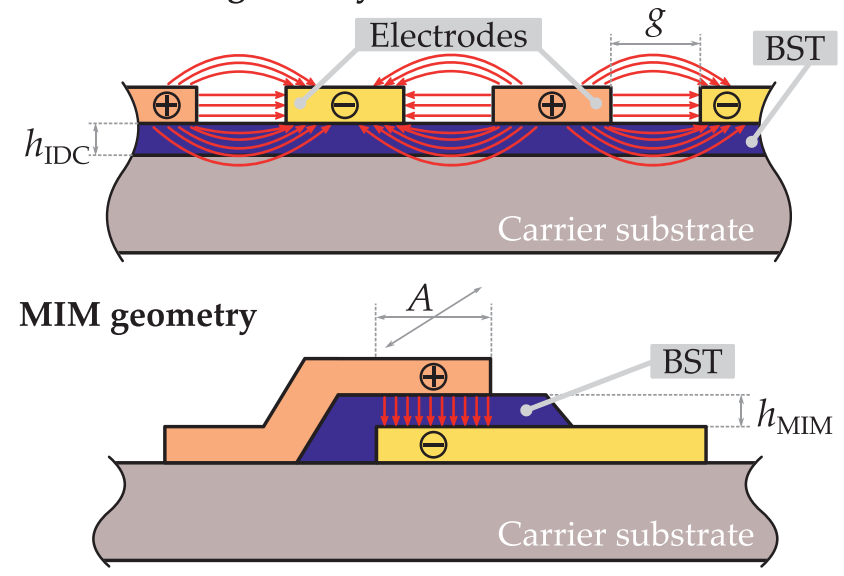

Figure 6: (Top) Planar (IDC) geometry of a BST varactor with critical dimensions of gap width $g$ and BST layer thickness $h_{\mathrm{IDC}}$. (Bottom) 3-D MIM structure with critical dimensions of area $A$ and BST layer thickness $h_{\text {MIM }}$.

efficiency of around $70 \%$ [9]. This drawback can be overcome with MIM structures, where the capacitance can be approximated by a parallel plate capacitor with area $A$ and distance $h_{\text {MIM }}$. For thick-film components, the MIM structures cannot take advantage of lower tuning voltages $\left(h_{\text {MIM }} \ll g\right)$ by saving a high tunability, because of lower material quality factor up to now. Higher losses are caused by compatible electrode materials with higher resistivity and lower material quality, resulting from the micro structure. For MIM thin-film components, the BST layer induces a piezoelectric effect to the component, which limits the application to low frequencies usually below $2 \mathrm{GHz}$ due to acoustic resonances [6].

\subsection{Liquid crystal (LC)}

During the last decade, liquid crystals (LC), well known from the display technology, have become increasingly popular in the field of microwave engineering [10], [11]. The name "liquid crystal" is a combination of the main properties of this material, since it is in a mesophase between a solid crystal and an isotropic liquid. The liquid crystalline mesophase is between the melting point of the crystalline phase and the clearing point to the isotropic liquid phase. In this mesophase, the LC is in the liquid phase, but shows properties usually seen for crystalline materials, esp. anisotropic material properties. Therefore, LC used for microwave applications can be described as uniaxial anisotropic material, featuring different effective permittivities, depending on the orientation to an applied $\mathrm{RF}$ field. Figure 7 shows a macroscopic representation of 


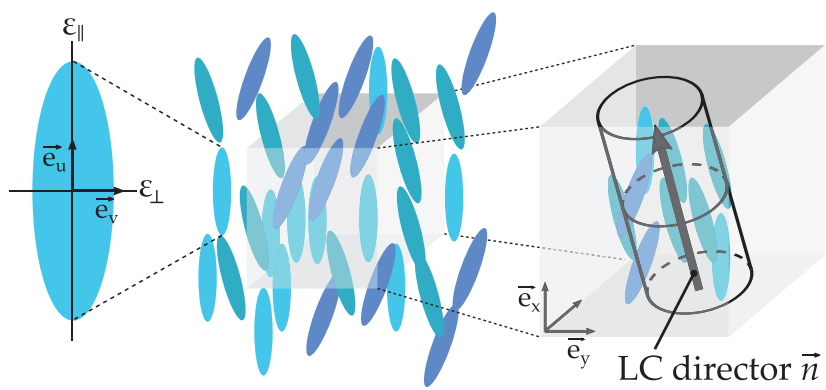

Figure 7: Definition of the director $\vec{n}$ for a single LC unit volume element.

the LC volume with the director $\vec{n}$, which describes the mean orientation of the LC molecules in a unit volume element. The effective permittivity of the LC material is depending on the orientation of the applied RF field $\vec{E}$ to the director $\vec{n}$. It can continuously take all values from the parallel permittivity $\varepsilon_{\|}$to the perpendicular permittivity $\varepsilon_{\perp}$ for $\vec{n} \propto \vec{E}$ and $\vec{n} \cdot \vec{E}=0$, respectively. A schematic representation of this principle in a hollow waveguide application can found in Figure 10.

As for ferroelectrics, the material performance of different liquid crystal mixtures can be evaluated based on the material performance $\eta_{\mathrm{LC}}$. It is based on the maximum dielectric losses, which usually occurs for the perpendicular state, and the anisotropy of the material by

$$
\eta_{L C}=\frac{\tau_{L C}}{\tan \delta_{\max }}=\frac{\varepsilon_{\|}-\varepsilon_{\perp}}{\varepsilon_{\|} \cdot \tan \delta_{\perp}} .
$$

Figure 8 shows the performance of different LC mixtures with LC used in display technology (such as K15 or E7) in the upper left with a material performance $\eta_{L C}>8.5$ at $19 \mathrm{GHz}$. Based on these basis mixtures and a close cooperation with Merck KGaA, Darmstadt, Germany, new mixtures could be developed, which show superior performance at microwave frequencies and sub-millimeter wave frequencies up to THz-frequencies [12].

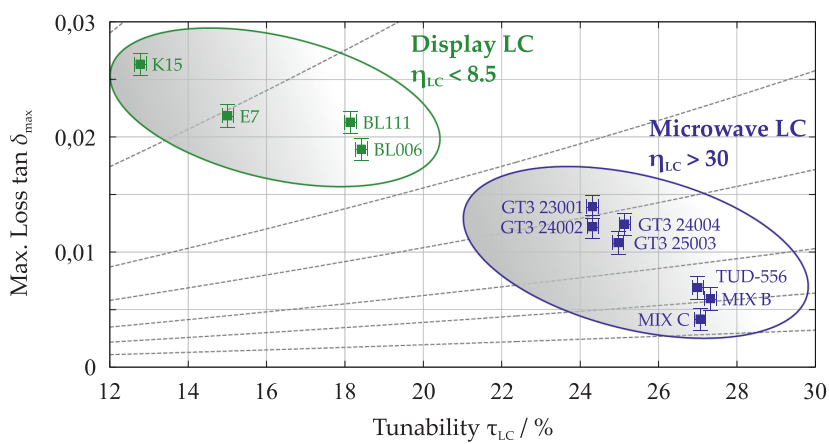

Figure 8: Performance of different LC mixtures at $19 \mathrm{GHz}$ The dashed lines represent constant material performance $\eta_{\mathrm{LC}}$.
The orientation of the LC molecules can be controlled by electric or magnetic fields and by mechanical anchoring at surfaces. For the first two cases the LC molecules tend to align parallel to the electric or magnetic field. Hence, by changing the orientation of a bias field the molecules can be aligned in regard to an electro-magnetic signal. For the third case, the LC molecules align parallel to the surface. In LCD technology also a perpendicular alignment by surface anchoring can alternatively be used. In tunable components only the parallel alignment is used, up to now. The final alignment of the LC is defined by the minimum of the Gibbs free energy influenced by the external fields, the surface anchoring and the material's elasticity parameters. Tunable components are usually based on transmission lines with tunable propagation constant. The simplest geometry is based on a microstrip line as shown in Figure 9. For applications, the LC volume has to be sealed from leaking. This can be realized by photolithographic patterned materials such as SU-8 or UV-hardened glue. Another, more sophisticated way is the integration in LTCC multilayer circuits, which contain embedded cavities for the LC. Another class of tunable transmission lines are hollow waveguides. Here a container filled with LC is inserted into the cross section of the wave-guiding structure, as shown in Figure 10. A new class of tunable transmission lines has been introduced by [13]. Here, a tunable dielectric
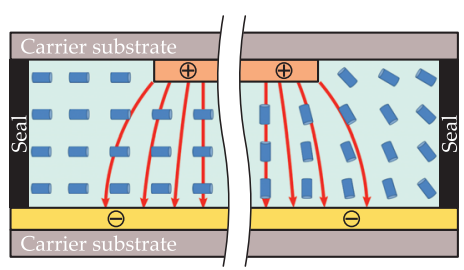

Figure 9: (Inverted) microstrip transmission line with LC as dielectric (left) in untuned state $\varepsilon=\varepsilon_{\perp}$ and (right) tuned state $\varepsilon \approx \varepsilon_{\|}$. The molecules are anchored at the surfaces by a rubbed polyimide film and are oriented by an electric field superimposed to the RF field.

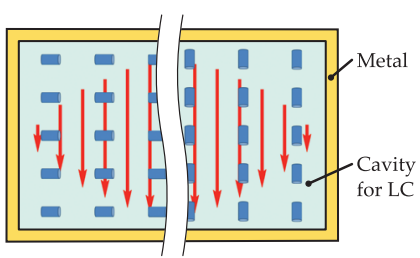

Figure 10: Hollow waveguide filled with LC (left) in idealized perpendicular and (right) parallel state and the TE10 mode. 


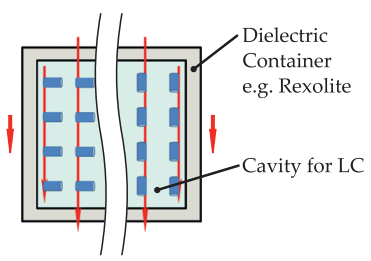

Figure 11: Dielectric sub-wavelength fiber waveguide partially filled with LC (left) in idealized perpendicular and (right) parallel state and the $E_{y}^{11}$.

fiber (Figure 11) has been used for the realization of a phase shifter in the W-band.

The material's tuning speed is dependent on the viscosity of the LC, the thickness of the LC layer and the applied tuning voltage. In case of surface anchoring without any external biasing, the forces within the LC are weak, resulting in long response times. Switching back from an electric field alignment to surface anchoring the time is proportional to $\tau_{\text {off }} \propto d^{2}$ with layer thickness $d$. This re-orientation process can easily take up to several seconds for a layer thickness of $100 \mu \mathrm{m}$. For electric field supported orientation (esp. from surface anchored to parallel to the field), the transition is much faster. The time can be approximated by $\tau_{\text {on }} \propto d^{2} / V_{B}^{2}$, but it can also be in the range of several seconds for large thickness. The only possibility for improving the response times of LC-based components, is a reduction of the LC layer thickness, which has been demonstrated by MIM varactors of few thickness [14].

Table I summarizes the properties of the different technologies for tunable microwave components.

\section{Applications in SatCom}

\subsection{Beam-steering phased-array antennas}

One possible application of such tunable components can be found in beam-steering antennas. Figure 12 shows the principle of a phased array antenna with tunable phase shifters at each antenna element fed by a common feeding network. For this simple example of a linear array, the far field of the antenna can be calculated by the element factor $\operatorname{EF}(\Theta)$ and the array factor $\operatorname{AF}(\Theta)$ by

$$
E(\Theta) \propto \mathrm{EF}(\Theta) \cdot \mathrm{AF}(\Theta)=\mathrm{EF}(\Theta) \cdot \sum_{n=0}^{N-1} e^{j n\left(k d \cos \Theta+\varphi_{n}\right)}
$$

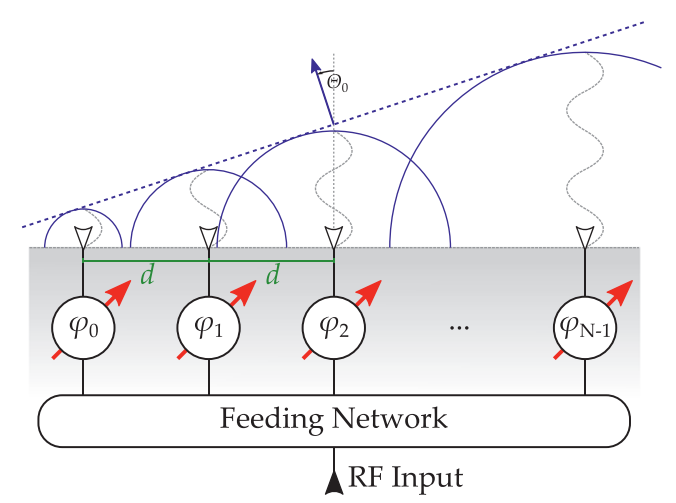

Figure 12: Schematic of a one dimensional phased array antenna.

Table 1: Technologies for tunable microwave components.

\begin{tabular}{|c|c|c|c|c|c|}
\hline & Semiconductor & Microsystems & Ferrites & Ferroelectrics & Liquid Crystal \\
\hline $\begin{array}{l}\text { Tuning of } \\
\text { Tuning by } \\
\text { Bias } \\
\text { Tuning speed } \\
\text { Linearity } \\
\text { Power consumption } \\
\text { Frequency / GHz }\end{array}$ & $\begin{array}{c}\text { Depletion zone } \\
\vec{E} \\
<5 \mathrm{~V} \\
\text { ps } \\
\text { Very low } \\
\text { High } \\
<5\end{array}$ & $\begin{array}{l}\text { Geometry } \\
\vec{E} \\
<200 \mathrm{~V} \\
\mu \text { s to ms } \\
\text { Moderate } \\
\quad \text { Low } \\
<300\end{array}$ & $\begin{array}{l}\stackrel{\vec{B}}{\text { Permeability }} \\
\text { Very high } \\
\text { Very high }\end{array}$ & $\begin{array}{c}\text { Permittivity } \\
\vec{E} \\
>200 \mathrm{~V} /<30 \mathrm{~V} \\
\text { ps } \\
\text { High / moderate } \\
\text { Low / high } \\
<15 /<3\end{array}$ & $\begin{array}{l}\text { Orientation } \\
\quad \vec{E} \\
<200 \mathrm{~V} \\
\text { s to min } \\
\text { High } \\
\text { Low } \\
>10\end{array}$ \\
\hline
\end{tabular}


with the phase offset $\varphi_{n}$ of each branch. Implementing tunable phase shifters in the array, the direction of the main beam $\Theta_{0}$ can easily by adapted to the needs by choosing the phase offset according to

$$
\varphi_{n}=n k d \sin \Theta_{0} .
$$

Two dimensional beam steering is also possible with the same concept by implementing a two dimensional array factor $\operatorname{AF}(\Theta, \Phi)$, e. g. by a two dimensional array antenna.

Therefore, tunable phase shifters are the main component of beam-steering antenna arrays. The figure of merit (FoM) for passive phase shifters (independent from the underlying technology) is defined as the maximum differential phase shift $\Delta \varphi_{\max }$ related to the maximum insertion loss $\mathrm{IL}_{\max }$ of all tuning states with

$$
\mathrm{FoM}=\frac{\Delta \varphi_{\max }}{\mathrm{IL}_{\max }} .
$$

Beside a high FoM, it is advantageous if the phase shifter exhibits a flat insertion loss profile over all tuning states to avoid additional amplitude tapering of the array.

BST based Phased Array Antennas: Figure 13 shows the layout of a left-handed transmission line-based BST phase shifter with the tunable varactors in the series branch of the transmission line. The bias voltage is fed through the shunt inductances to the varactors. The phase shifter shows a phase shift of more than $360^{\circ}$ in the designed frequency band from $6 \mathrm{GHz}$ to $11 \mathrm{GHz}$ resulting in a FoM of around $55^{\circ} / \mathrm{dB}$ The phase shifter has been incorporated into a $1 \times 4$ array antenna system as shown in Figure 14. More details on the fabrication and performance of the antenna can be found in [15]. For the

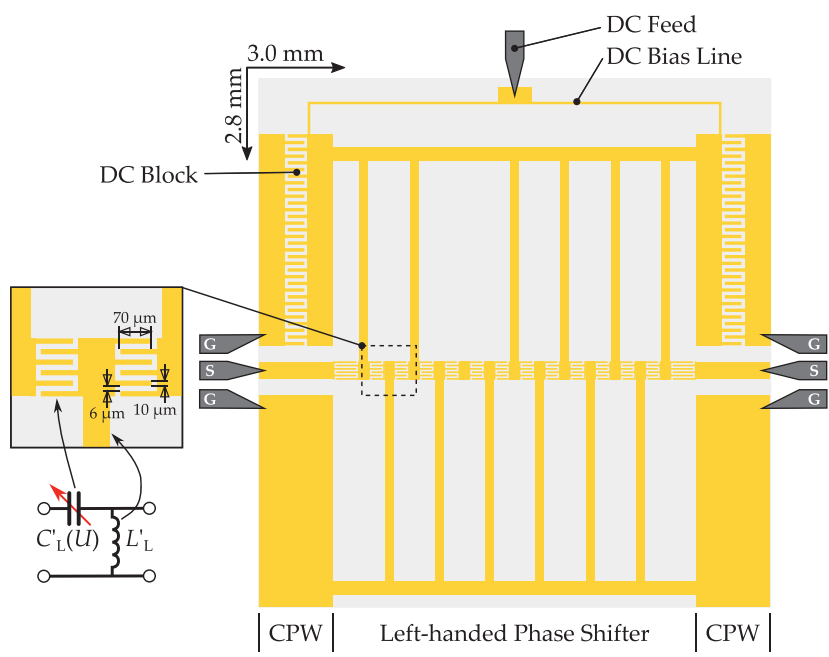

Figure 13: Layout of a BST phase shifter using a left handed transmission line. implementation, the phase shifter has to be combined with a microstrip to coplanar waveguide transition. The reason is, that based on the used thick film technology only one patterned layer can be realized, due to the sintering step of the BST thick film at $1,200^{\circ} \mathrm{C}$. Another drawback of this technology are increased losses due to the full face printing of the BST in areas where no functionality is required, e. g. below the patch antenna elements and the feeding network. As already mentioned, there are different technologies available to overcome this issue. One possibility is a selective screen printing of the BST only to areas which require the functionality. Figure 15 shows the realization of such a phase shifter based on a periodically capacitively loaded transmission line, where the BST has been selectively printed only in the areas where needed by means of ink-jet printing [7]. This is made possible by a low temperature sintering BST thick film, which enables the realization of a multilayer structure with a MIM configuration of the BST varactors between two photolithographically patterned bottom and top electrodes. These phase shifters for $8 \mathrm{GHz}$ show with $44 \% \mathrm{~dB}$ a little lower FoM than for fully printed BST thick film phase shifters. This is due to a little lower material performance of the printed BST thick film, which shows slightly poorer performance. Based on this, a $1 \times 4$ array antenna has been realized. The antenna elements are realized by aperture coupled dielectric resonators [16]. Figure 16 shows the realization of this antenna. The whole system consists of an Alumina substrate with the phase shifters and a Rogers R04003C PCB for the ground plane and the aperture coupling to the dielectric resonators. The antenna shows a beam steering of $\pm 30^{\circ}$ with an estimated gain of $13 \mathrm{dBi}$.

A possible next step are fully printed phase shifters, where also the metallic electrodes are printed by an ink jet printer. In [17] the design of the phase shifters described above has been reused with the varactors replaced by a fully ink jet printed version. Figure 17 shows a picture of a single varactor fabricated in three layer ink jet printing with sintering after every layer. The bottom and top layers are mode of an LTCC compatible commercial silver paste (TC 7306 A, Heraeus, Hanau, Germany). The BST layer in between is a low temperature sintering copper-fluorine co-doped BST with 20 vol\%. of $\mathrm{ZnO}$ and $\mathrm{H}_{3} \mathrm{BO}_{3}$ with a sintering temperature of around $850^{\circ} \mathrm{C}$. Due to much lower material quality, the performance of the fabricated phase shifters is again lower than for previous technologies. The FoM reaches values around $37 \% \mathrm{~dB}$ at $3 \mathrm{GHz}$. This technology is at the very beginning, offering many opportunities for improvement. On the one hand, the printed materials have to be 


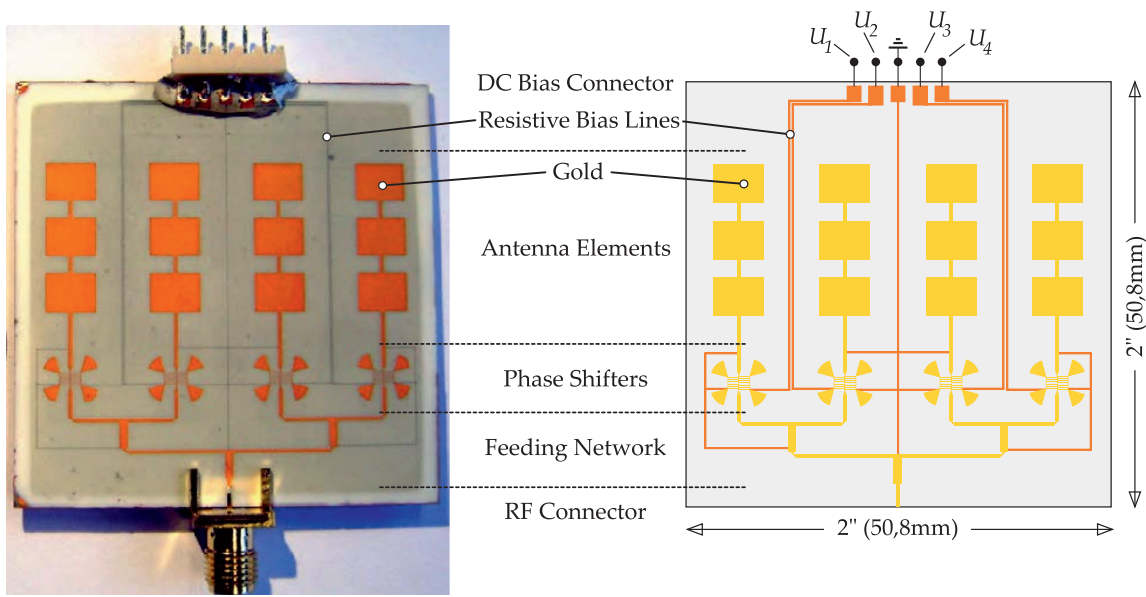

Figure 14: (Left) Photograph and (right) schematic of a BST-based $1 \times 4$ phased array antenna with a $3 \times 1$ sub-array configuration. An SMA and a five-pin connector are used for RF and DC bias supply, respectively. The metallized backside is used as ground plane.

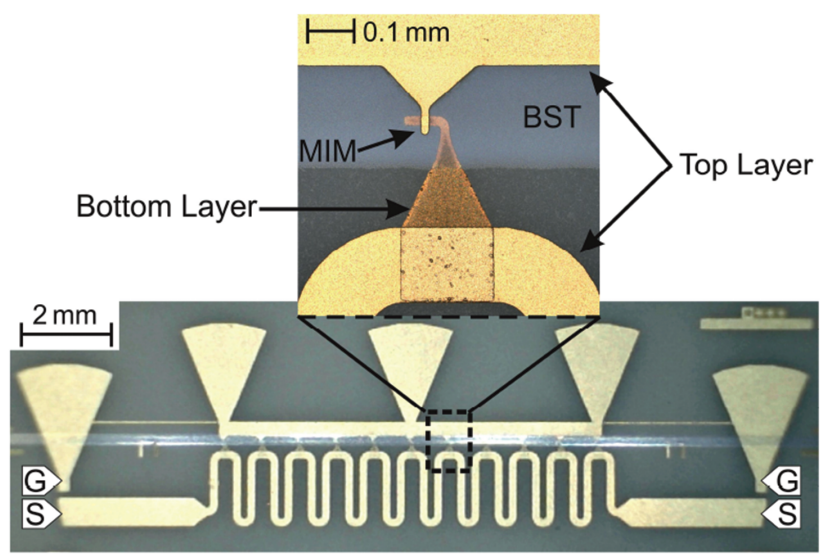

Figure 15: Photograph of a loaded line phase shifter with MIM varactors based on an ink-jet printed BST thick film.

optimized, since the BST but also the silver inks cannot compete with screen printed and electroplated materials. On the other hand, the resolution of the printers in combination with the high permittivity of the BST layers makes it very difficult realizing components with small capacitance values and low tuning voltages. The thickness of the printed BST layer has a thickness of $5 \mu \mathrm{m}$, which requires tuning voltages of up to $200 \mathrm{~V}$, which is

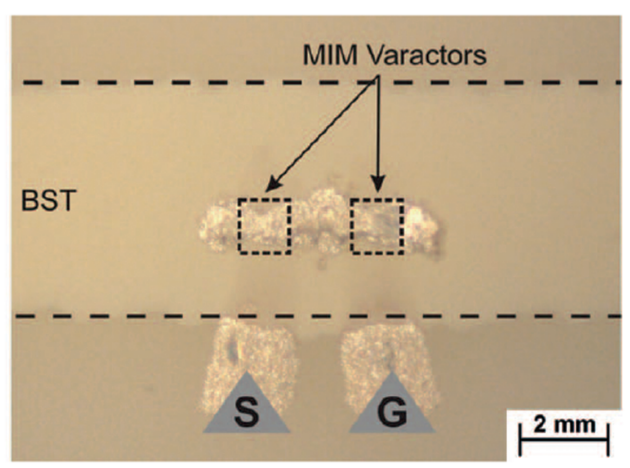

Figure 17: Photograph of the fully ink jet printed BST varactor.

comparable to standard planar IDC-based varactors on screen printed thick film.

In this section three different approaches have been presented for the realization of beam-steering phasedarray antennas based on BST thick film technology. Table 2 summarizes the results from previously shown examples with typical values for the underlying technology. The standard screen printing technology offers the best performance over a wide frequency range. Here, phase shifters with high FoM can be realized. The inkjet
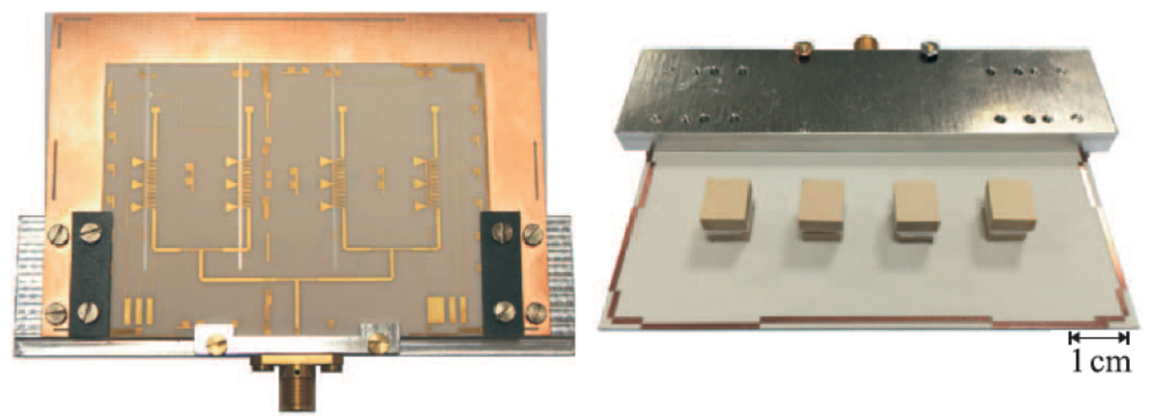

Figure 16: Photograph of a $1 \times 4$ phased array antenna with ink-jet printed BST phase shifters and dielectric resonator based radiators. 
Table 2: Comparison of different BST thick film processing technologies with typical values.

\begin{tabular}{llll}
\hline & $\begin{array}{l}\text { Screen } \\
\text { printed }\end{array}$ & $\begin{array}{l}\text { Inkjet } \\
\text { printed }\end{array}$ & $\begin{array}{l}\text { Fully ink jet } \\
\text { printed }\end{array}$ \\
\hline BST & $\begin{array}{l}\text { Screen } \\
\text { printed }\end{array}$ & $\begin{array}{l}\text { Ink jet } \\
\text { printed }\end{array}$ & Ink jet printed \\
Electrodes & Electroplated & Electroplated & Ink jet printed \\
Topology & IDC & MIM & MIM \\
Quality (Losses) & High & Medium & Low \\
Electrode distance & $5 \mu \mathrm{m}$ to $20 \mu \mathrm{m}$ & $1 \mu \mathrm{m}$ to $2 \mu \mathrm{m}$ & $4 \mu \mathrm{m}$ to $6 \mu \mathrm{m}$ \\
Tuning voltage & $200 \mathrm{~V}$ & $50 \mathrm{~V}$ & $100 \mathrm{~V}$ \\
Tunability & $50 \%$ & $50 \%$ & $33 \%$ \\
Frequency range & $\leq 15 \mathrm{GHz}$ & $\leq 8 \mathrm{GHz}$ & $\leq 5 \mathrm{GHz}$ \\
\hline
\end{tabular}

printing technologies offer many new fields of application and realization approaches. Up to now, this technology lacks in performance, especially of the BST film's and electrodes' quality, resulting in higher losses compared to standard screen printing. Nevertheless, the progress of recent research promises to come close to established technologies.

LC based Phased Array Antennas:Liquid crystal based phase shifters show superior performance at high frequencies. The main design challenges are the confinement of the liquid inside the cavity and the implementation of the biasing structures. Figure 18 shows the realization of a hollow waveguide based phase shifter for the $\mathrm{K}_{a}$-band [18]. The phase shifter is designed in a split-block topology, surrounding the LC filled container with its

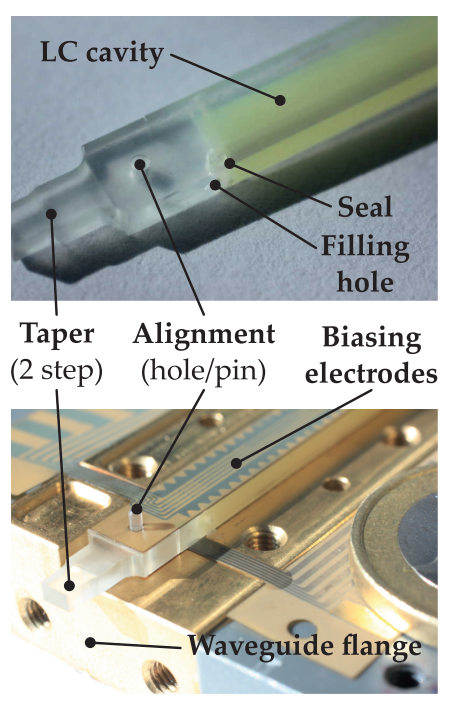

Figure 18: Photographs of (top) the dielectric tapering of the Rexolite container including the LC cavity as well as alignment and filling hole, and (bottom) the assembled phase shifter without the upper split-block lid, including the LC filled Rexolite container and the biasing structures. biasing electrodes. The container is made of Rexolite 1422 $\left(\varepsilon_{r}=2.53, \tan \delta=0.66 e-3\right.$ at $\left.10 \mathrm{GHz}\right)$, a space qualified, hard plastic material. The phase shifter takes advantage out of the fact that the $\mathrm{TE}_{10}$ mode has the maximum field in the center of the hollow waveguide. A partial filling of the cross section of the waveguide results in a lower tuning efficiency, but at the same time the insertion loss decreases due to higher material quality. In addition with the properties of LC, the wave is focused in the low loss case of the tuning state $\left(\varepsilon_{\|}\right)$in the LC due to the higher permittivity. In the high loss case $\left(\varepsilon_{\perp}\right)$ the energy can be spread more out into a container with low dielectric loss. With this, the overall performance of the phase shifter is increased. The differential phase shift reaches $460^{\circ}$ with a very high FoM of approximately $130 \% \mathrm{~dB}$. The biasing structures are realized on a Mylar film with $50 \mu \mathrm{m}$ thickness. It is based on a quadrupole design [18]-[20] in order to generate the different biasing fields required for aligning the LC. The LC is aligned by electric forces only, because the surface anchoring is not applicable. Response time measurements show maximum switching times from one state to the other between one to two minutes. The phase shifter is designed in a way that it can be implemented into the feeding network of a satellite antenna, which is planned to be launched on the Heinrich-Hertz-Mission from DLR. Figure 19 shows the final design of the phase shifter with low weight electroplated waveguide walls. Combined with two light-weight aluminum flanges, this phase shifter has a weight of below $25 \mathrm{~g}$. The phased horn array could not be characterized yet, as the fabrication of the phase shifters turned out to be more difficult than anticipated. However, from phase shifter measurements

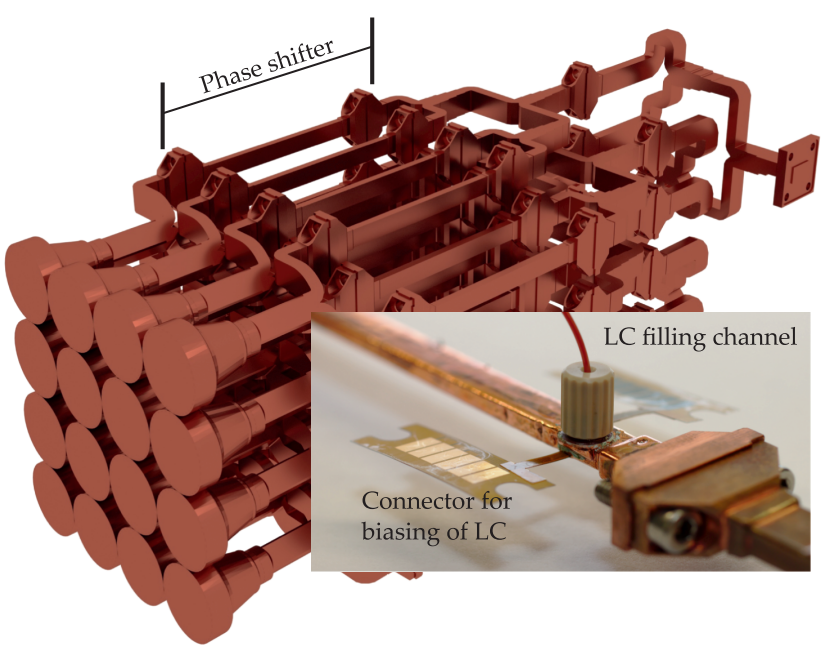

Figure 19: CAD model of the horn antenna array of the LISA project with the intended place of the phase shifter in the feeding network. The inset shows the picture of the final phase shifter with the connector pads for the biasing structure. 
combined with earlier measurements of the static antenna array, it can be concluded, that the performance specifications can be met: The steering range is a cone of $\pm 11^{\circ}$, satellites with an apparent angular speed as seen from GEO of $0.8 \% \mathrm{~min}$ can be tracked [21].

The design of hollow waveguide phase shifters gets more complicated for higher frequencies. Not only the size of the containers get smaller and more difficult to process, also the size of the biasing electrodes has to be adapted to the smaller size close to the resolution limit of conventional lithographic processes. Although first designs of LC based phase shifters have been presented at $250 \mathrm{GHz}$ [22]. A new technology shows much higher potential, especially at higher frequencies. In [13] the first implementation of a tunable dielectric sub-wavelength fiber based on LC is presented. The fiber in Figure 20 consists of Rexolite, because of its good workability, stability, low dielectric loss and matching permittivity. In the center of the cylindrical Rexolite fiber with a diameter of $2 \mathrm{~mm}$, a cavity for the LC is drilled with a diameter of $0.6 \mathrm{~mm}$ and a total length of $26 \mathrm{~mm}$ for the tunable line segment. For proof of concept, the phase shifter has been magnetically biased. In a second step, an electrical biasing system has been built. Two pairs of parallel plate electrodes surrounding the fiber are forming the electrical biasing system. The maximum biasing voltage was $\pm 500 \mathrm{~V}$. Such high voltages are needed, since the biasing electrodes need to be separated sufficiently far from each other not to disturb the evanescent field components. This phase shifter reaches in the first design a FoM of around $45 \% \mathrm{~dB}$ in the entire $\mathrm{W}$-band $(75 \mathrm{GHz}$ to $110 \mathrm{GHz}$ ). Based on this design array antennas and more complex integrated systems can be realized. One possible application beside array antennas is the realization of single-pole double-throw (SPDT) switches, e. g. to switch between calibration loads and the antenna of a radiometer.

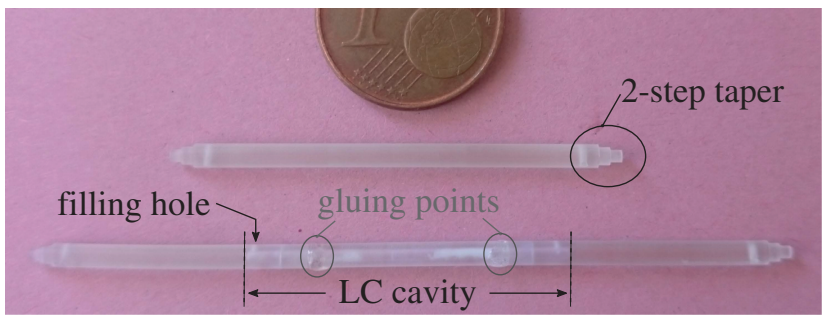

Figure 20: Photograph of the sub-wavelength dielectric fiber phase shifter (bottom) in comparison with an untunable fiber (top) with the same electrical length. For measurement purposes the transmission lines are tapered to fit into a WR-10 adapter for measurements. The LC cavity is highlighted for better visibility.
In [23] a 2D-steerable planar array based on a technology similar to that of LC displays has been presented. This design is based on the (inverted) microstrip line technology. It uses two glass substrates for the signal lines. The LC cavity is formed between the glasses by spacer pearls with a diameter of approx. $100 \mu \mathrm{m}$. The phase shifters operate in the $\mathrm{K}_{\mathrm{u}}$ band, offering a FoM of more than $50 \% \mathrm{~dB}$ from $13 \mathrm{GHz}$ to $19.5 \mathrm{GHz}$. The early demonstrator, shown in Figure 21, offers a beam steering of $\pm 25^{\circ}$ in both $\mathrm{E}$ - and $\mathrm{H}$-plane. Based on this design also larger array antennas can be built. Figure 22 shows the realization of an $8 \times 8$ array antenna of $12 \mathrm{~cm} \times 12 \mathrm{~cm}$ size. The main advantage of this type is the thickness of less than $2 \mathrm{~mm}$ as well as the scalability for larger arrays. Simulations have shown that a $32 \times 32$ array antenna built with this technology shows a gain of more than $25 \mathrm{~dB}$ and a grating lobe free region up to $\pm 55^{\circ}$ of beam

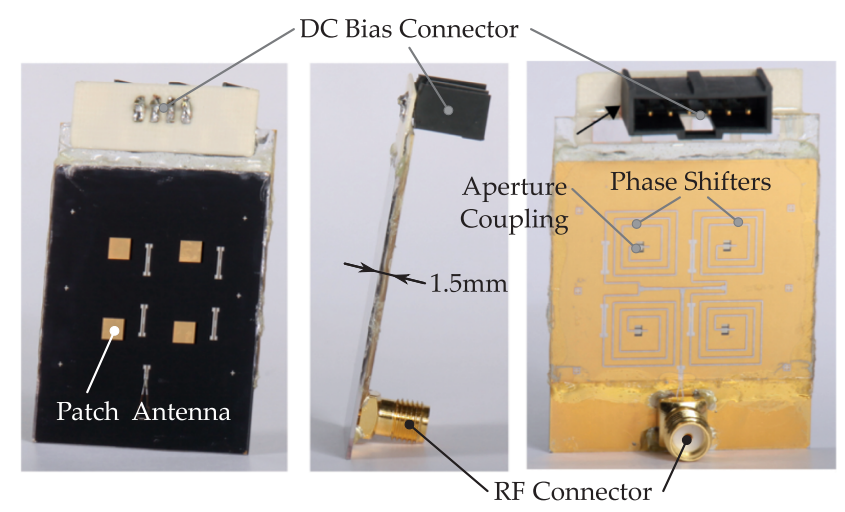

Figure 21: 2D-steerable planar $2 \times 2$ array with a thickness of $1.5 \mathrm{~mm}$.

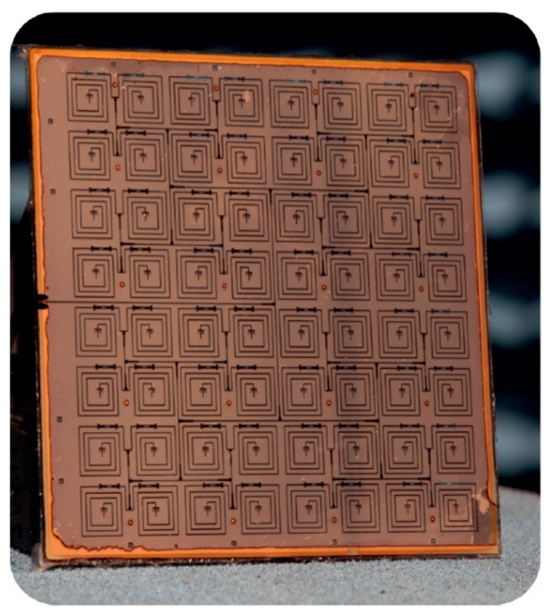

Figure 22: Backside view of the $8 \times 8$ antenna array with the 64 individual meandered phase shifters and the feeding network. On the backside of the $1.5 \mathrm{~mm}$ thick substrate 64 aperture coupled patch elements are realized. 
steering. Meanwhile, this antenna concept has been commercialized. The planar antenna technology and the antennas will be advanced by the company ALCAN Systems, Darmstadt, Germany.

\subsection{Tunable filters}

Beside phase shifters, tunable filters are of major interest especially for satellite operators. Heavy and bulky switched filter banks are replaced by a single tunable filter with adaptable center frequency and/or bandwidth. Filters require even higher performance of the tunable components in terms of tunability and losses compared to phase shifters.

BST-based Tunable Filters: One possible application of tunable filters is in the IF band of transceiver frontends. Due to the relatively low frequency comparable to terrestrial mobile communication systems, BST based and/or semiconductor based tunable filters can be implemented. Figure 23 shows the realization of a BST thick film based tunable IF band filter. This early demonstrator realization is a filter of order three with a hybrid integration of screen printed BST MIM varactors and discrete inductors [24]. The simulation results are summarized in Figure 24. The Filter shows a tuning of the center frequency and at the same time of the filter bandwidth, due to the tunable coupling between the resonators. The DC decoupling of the varactors is realized with $1 \mathrm{M} \Omega$ resistors. The tunability of the used varactors is around $50 \%$ to $60 \%$ with a bias voltage of $400 \mathrm{~V}$. The sum of all leakage currents $\leq 1 \mu \mathrm{A}$ makes the power consumption of the filter negligible. The third order output intercept point OIP3 has been measured at $61 \mathrm{dBm}$ proving the

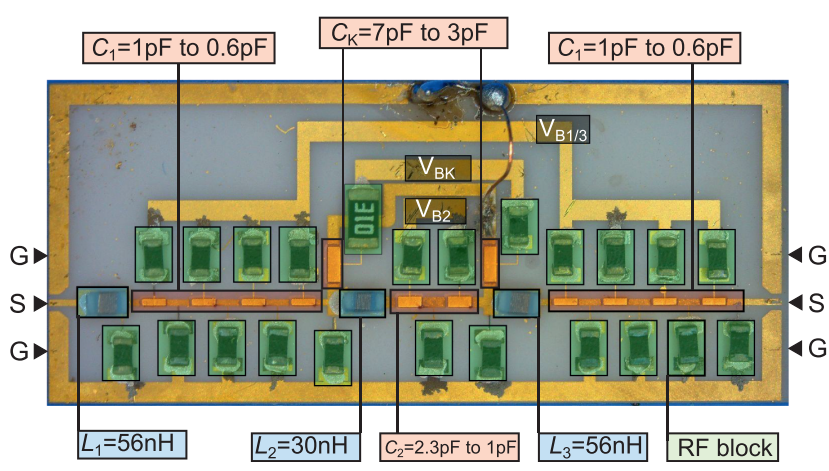

Figure 23: Layout of a BST thick film based filter for IF band applications. The blue shaded areas denote the SMD inductors, the red shaded areas denote all the tunable BST-based varactors while the green areas mark the $1 \mathrm{M} \Omega$ RF block resistors.
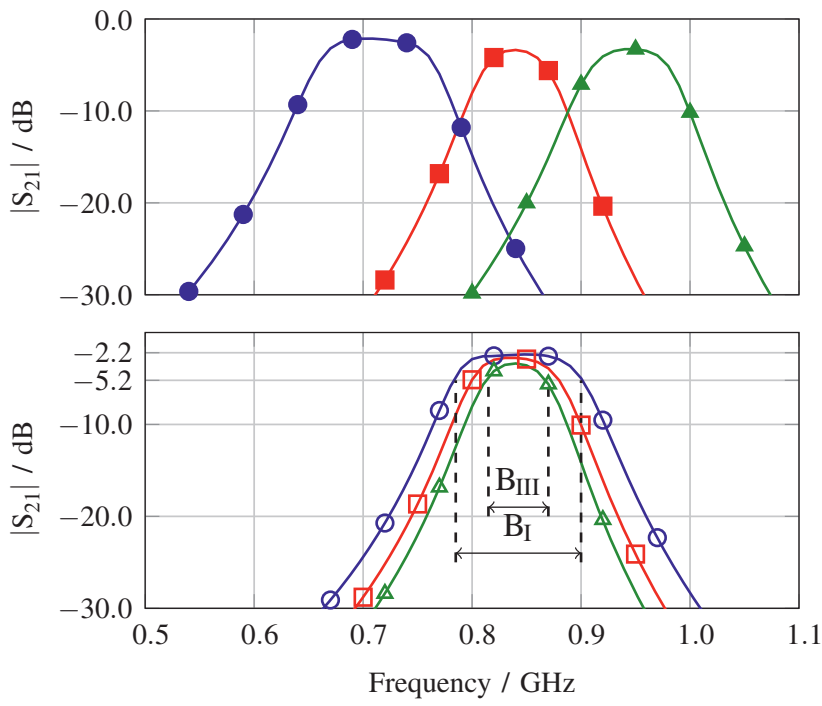

Figure 24: Simulation results of the BST thick film based IF filter. (Top) tuning of the center frequency (bottom) tuning of the bandwidth.

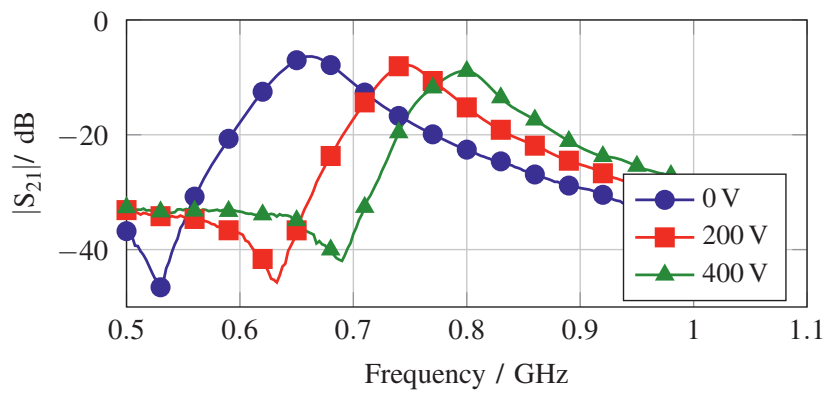

Figure 25: Measurement results of the BST thick film based IF filter for tuning of the center frequency.

high linearity of BST thick film varactors. The realized filter shows tuning of the center frequency from $650 \mathrm{MHz}$ to $800 \mathrm{MHz}$ with an insertion loss of 6 to $9 \mathrm{~dB}$, compare Figure 25. Further analysis of the results of this early demonstrator indicate that the poor performance is mainly caused by the fabrication tolerances of the BST varactors. It becomes apparent that the poles are pushed apart at the low limit of the DC bias voltage of the coupling shunt varactors $C_{K}$. Furthermore, with increasing DC bias voltage one pole is shifted to higher frequencies, consequently further pushing the poles apart. Furthermore, this results in worse impedance matching compared to the simulation and prevents the measurement of the bandwidth tunability. Nevertheless, the work on BST tunable filters shows a great potential. Future work will include, beside the optimization, a systematic comparison with other technologies such as BST thin film varactors and semiconductor varactors. 
LC based Tunable Filter: Much higher filter qualities can be achieved in LC tuned cavity filters at higher frequencies. The first lab demonstrator of a tunable 3-pole filter is based on a radially coupled cylindrically resonator operating in the $\mathrm{TM}_{010}$ mode [25], [26]. The core component of the tunable resonators is an LC filled, pillbox-shaped container made of Rexolite. Here, contrary to the phase shifter, the top of the Rexolite container is glued after the LC filling. This brings the advantage of a perfect sealing but accompanied with the drawback of a lossy glue inside the resonator. Similar to the phase shifter, the Rexolite provides only a cavity of the LC in the center of the resonator, where the RF field is strongest, see also Figure 26. This affects the tuning range only a little and comes with the advantage of lower insertion loss due to the less lossy Rexolite cladding. The electric biasing system is implemented in a LTCC multilayer structure. The main disadvantage for space applications of this layout is its sensitivity to temperature changes. Due to the higher thermal expansion coefficient of LC compared to Rexolite, the container can burst under high temperature changes. A possible solution is to use another design, which is based on a radially inserted dielectric rod filled with LC, compare Figure 27. This design offers the possibility of a pressure compensation by installing a flexible bellow system outside the resonator, in order to compensate for volume changes of the LC. An additional advantage is the absence of a lossy glue inside the resonator. Nevertheless, the tuning range will be smaller compared to the pillbox-shaped container since there is less LC in the center of the resonator, due to the limited rod diameter. For investigating the RF-performance of the different LC cavity topologies, single resonators were fabricated. For the pillbox-shaped container an unloaded Q-factor of $Q=484$ and $Q=327$ at a resonance frequency of $f=20.17 \mathrm{GHz}$ and $f=19.77 \mathrm{GHz}$ is measured for the parallel and perpendicular tuning state of the LC, respectively. For these measurements magnetic biasing is used. The rod-shaped resonator design shows lower $\mathrm{Q}$ factors of $Q=256$ and $Q=170$ for a resonance frequency of $f=23.49 \mathrm{GHz}$ and $f=23.98 \mathrm{GHz}$. Further, a 3-pole filter with pillbox-shaped LC containers is realized and characterized using magnetic biasing. The results are presented in Figure 28. The intended tuning range is not reached, but a tuning range of $450 \mathrm{MHz}$ could be
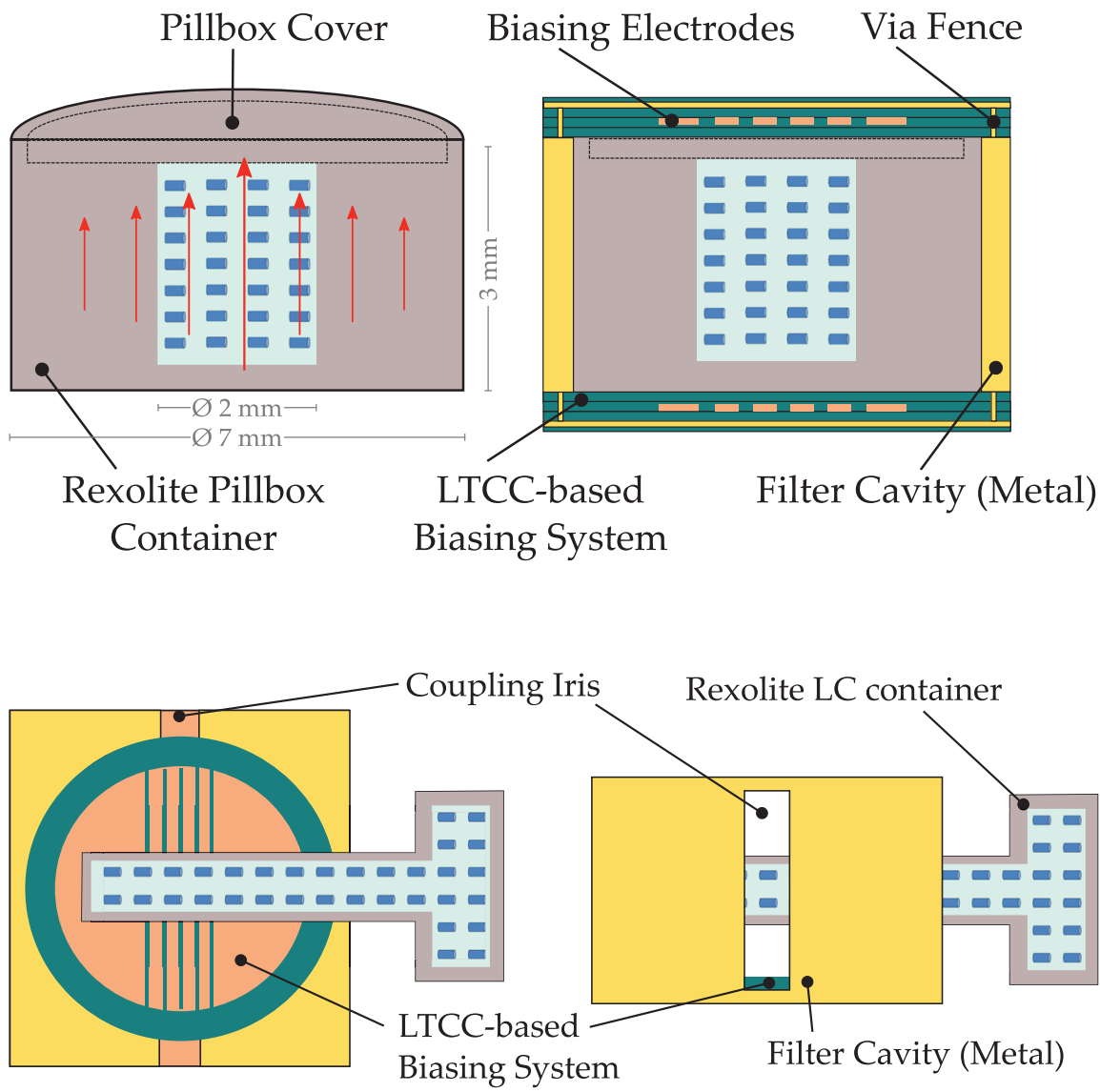

Figure 26: Schematic cross section (left) of the pill-shaped Rexolite container including the $\mathrm{LC}$ and showing the $\mathrm{TM}_{010}$ mode given by the arrows, and (right) of the complete resonator structure, including the metallic walls and the electric biasing system.
Figure 27: Schematic (left) cross section of the top view of the resonator including the rod-shaped LC cavity and (right) front view with an inter-resonator coupling iris. 


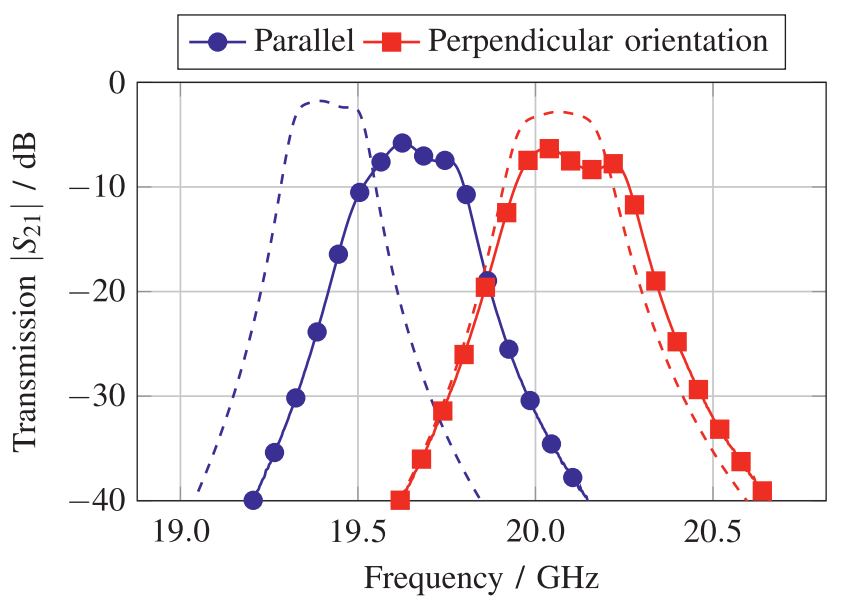

Figure 28: Simulation and measurement results of the pillbox-based 3-pole filter using magnetic biasing. The simulations (dashed lines) were carried out using CST Studio Suite.

achieved. This originates from residues of glue and small air bubbles in the LC cavity. Despite the air bubbles, the frequency of the perpendicular state is not changing, since it is compensated by a further fabrication tolerance, a gap between the waveguide walls and the cavity. The shape of the curves is slightly detuned compared to the simulations but nevertheless, the losses over the different tuning states stay nearly constant.

\section{Conclusion}

In this paper, we presented different technologies for tunable components for SatCom applications. Table 1 summarizes their properties. While semiconductors can be monolithically integrated into circuits, they do not offer sufficient performance for most applications due to their main limitation in frequency range, required tuning power, and linearity. Here, alternative technologies such as ferroelectrics and liquid crystal are preferred options. Both technologies show similar behavior in their frequency bands. For SatCom applications ferroelectrics are suitable in the IF frequency bands while LCs are best suited at the antenna of the RF frontend, e.g. in reconfigurable array antenna systems.

Acknowledgment: The authors acknowledge the cooperation partners at KIT IAM-KWT, TUM LRT, DLR e.V., BAM, Merck KGaA, CST AG, EADS Astrium, Tesat, IMST $\mathrm{GmbH}$, NTP GmbH, and LOEWE STT as well as DFG for providing the platform for parts of this work.

\section{References}

[1] Die Zukunft der Satellitenkommunikation - Ein Weißbuch der Informationstechnischen Gesellschaft im VDE (ITG). 1em plus 0.5em minus 0.4em VDE ITG, 2016.

[2] S. Heinen and R. Wunderlich, "High dynamic range RF frontends from multiband multistandard to cognitive radio," in Semiconductor Conference Dresden (SCD), Sep. 2011, pp. 1-8.

[3] O. G. Vendik and S. P. Zubko, "Ferroelectric phase transition and maximum dielectric permittivity of displacement type ferroelectrics (BaxSr1-xTiO3)," J. Appl. Phys., vol. 88, no. 9, pp. 5343-5350, 2000.

[4] X. Zhou, H. Geßwein, M. Sazegar, A. Giere, F. Paul, R. Jakoby, J. Binder, and J. Haußelt, "Characterization of metal (Fe, Co, Ni, $\mathrm{Cu}$ ) and fluorine codoped barium strontium titanate thick-films for microwave applications," J. Electroceram., vol. 24, no. 4, pp. 345-354, 2010.

[5] C. Kohler, A. Friederich, M. Sazegar, M. Nikfalazar, F. Stemme, D. Wang, C. Kuebel, R. Jakoby, and J. Binder, "Effects of ZnO-B2O3 addition on the microstructure and microwave properties of low-temperature sintered barium strontium titanate (BST) thick films," Int. J. Appl. Ceram. Technol., vol. 10, pp. E200-E209, 2013.

[6] S. Gevorgian, A. Vorobiev, and T. Lewin, "dc field and temperature dependent acoustic resonances in parallel-plate capacitors based on $\mathrm{SrTiO}_{3}$ and ba0.25sr0.75TiO3 films: Experiment and modeling," J. Appl. Phys., vol. 99, no. 12, p. 124112, Jun. 2006.

[7] M. Nikfalazar, A. Mehmood, M. Sohrabi, A. Wiens, Y. Zheng, H. Maune, R. Jakoby, M. Mikolajek, A. Friederich, C. Kohler, and J. Binder, "Low bias voltage tunable phase shifter based on inkjet-printed BST MIM varactors for C/X-band phased arrays," in European Microwave Conference (EuMC), Sep. 2015.

[8] S. Gevorgian, H. Berg, H. Jacobsson, and T. Lewin, "Application notes - basic parameters of coplanar-strip waveguides on multilayer dielectric/semiconductor substrates, part 1: High permittivity superstrates," IEEE Microwave Mag., vol. 4, no. 2, pp. 60-70, Jun. 2003.

[9] H. Maune, M. Sazegar, Y. Zheng, X. Zhou, A. Giere, P. Scheele, F. Paul, J. R. Binder, and R. Jakoby, "Nonlinear ceramics for tunable microwave devices part II: RF-characteriziation and component design," Microsyst. Technol., vol. 17, no. 2, pp. 213-224, 2011.

[10] C. Weil and R. Jakoby, "Nonlinear dielectrics for microwave applications-ferroelectric and liquid crystals," IEEE MTT/AP Ger. Newslett., vol. 6, pp. 2-7, 2002.

[11] C. Weil, G. Luessem, and R. Jakoby, "Tunable inverted-microstrip phase shifter device using nematic liquid crystals," in IEEE MTT-S International Microwave Symposium, vol. 1, Jun. 2002, pp. 367-371.

[12] C. Weickhmann, R. Jakoby, E. Constable, and R. A. Lewis, "Timedomain spectroscopy of novel nematic liquid crystals in the terahertz range," in International Conference on Infrared, Millimeter, and Terahertz Waves (IRMMW-THz), Sept 2013, pp. 1-2.

[13] M. Jost, R. Reese, C. Weickhmann, C. Schuster, O. Karabey, H. Maune, and R. Jakoby, "Tunable dielectric delay line phase shifter based on liquid crystal technology for a SPDT in a radiometer calibration scheme at $100 \mathrm{GHz}$," in IEEE MTT-S Symposium, 2016. 
[14] F. Goelden, A. Gaebler, S. Mueller, A. Lapanik, W. Haase, and R. Jakoby, "Liquid-crystal varactors with fast switching times for microwave applications," Electron. Lett., vol. 44, pp. 480-481, Mar. 2008.

[15] M. Sazegar, Y. Zheng, H. Maune, C. Damm, X. Zhou, J. Binder, and R. Jakoby, "Low-cost phased-array antenna using compact tunable phase shifters based on ferroelectric ceramics," IEEE Trans. Microwave Theory Tech., vol. 59, no. 5, pp. 1265-1273, 2011.

[16] M. Nikfalazar, A. Mehmood, M. Sohrabi, M. Mikolajek, A. Wiens, H. Maune, C. Kohler, J. Binder, and R. Jakoby, "Steerable dielectric resonator phased array antenna based on inkjet-printed tunable phase shifter with BST metalinsulator-metal varactors," IEEE Antennas Wireless Propag. Lett., vol. 15, pp. 877-880, 2016.

[17] M. Nikfalazar, C. Kohler, A. Wiens, A. Mehmood, M. Sohrabi, H. Maune, J. Binder, and R. Jakoby, "Beam steering phased array antenna with fully printed phase shifters based on low-temperature sintered BST-composite thick films," IEEE Microwave Wireless Compon. Lett., vol. 26, no. 1, pp. 70-72, Jan. 2016.

[18] C. Weickhmann, N. Nathrath, R. Gehring, A. Gaebler, M. Jost, and R. Jakoby, "A light-weight tunable liquid crystal phase shifter for an efficient phased array antenna," in European Microwave Conference (EuMC), Oct. 2013, pp. 428-431.

[19] A. Gaebler, F. Goelden, A. Manabe, M. Goebel, S. Mueller, and R. Jakoby, "Investigation of high performance transmission line phase shifters based on liquid crystal," in European Microwave Conference, Sept. 2009, pp. 594-597.

[20] A. E. Prasetiadi, S. Rahmawati, C. Weickhmann, M. Nickel, M. Jost, T. Franke, W. Hu, H. Maune, and R. Jakoby, "Electrical biasing scheme for liquid-crystal-based tunable substrate integrated waveguide structures," in German Microwave Conference (GeMiC), Mar. 2016, pp. 136-139.

[21] M. Tebbe, A. Hoehl, N. Nathrath, and C. Weickhmann, "Simulation of an electronically steerable horn antenna array with liquid crystal phase shifters," in IEEE Aerospace Conference, 2016.

[22] C. Weickhmann, M. Jost, D. Laemmle, and R. Jakoby, "Design and fabrication considerations for a $250 \mathrm{GHz}$ liquid crystal phase shifter," in International Conference on Infrared, Millimeter, and Terahertz waves (IRMMW-THz), 2014.

[23] O. H. Karabey, A. Gaebler, S. Strunck, and R. Jakoby, "A 2-D electronically steered phased-array antenna with $2 \times 2$ elements in LC display technology," IEEE Trans. Microwave Theory Tech., vol. 60, no. 5, pp. 1297-1306, May 2012.

[24] C. Schuster, A. Wiens, M. Schößler, R. Jakoby, C. Kohler, and J. R. Binder, "Tunable lumped-element-filter for RF power applications based on printed ferroelectrics," in German Microwave Conference (GeMiC), Mar. 2016, pp. 229-232.

[25] R. Follmann, D. Köther, M. A. Campo, T. Franke, A. Gabler, R. Jäkoby, A. Manabe, T. Rabe, A. Heunisch, A. Rauch, and T. Kässer, "Liquida-sky: a tunable liquid crystal filter for space applications," in IEEE-APS Topical Conference on Antennas and Propagation in Wireless Communications (APWC), Sep. 2013, pp. 90-93.

[26] T. Franke, A. Gaebler, A. E. Prasetiadi, and R. Jakoby, "Tunable Ka-band waveguide resonators and a small band band-pass filter based on liquid crystals," in European Microwave Conference, Oct. 2014, pp. 339-342. 